PROCEEDINGS OF THE

AMERICAN MATHEMATICAL SOCIETY

Volume 138, Number 2, February 2010, Pages 703-715

S 0002-9939(09)10105-3

Article electronically published on October 7, 2009

\title{
SOME REMARKS ON THE POINCARÉ-BIRKHOFF THEOREM
}

\author{
PATRICE LE CALVEZ AND JIAN WANG
}

(Communicated by Bryna Kra)

\begin{abstract}
We define the notion of a positive path of a homeomorphism of a topological space. It seems to be a natural object to understand Birkhoff's arguments in his proof of the celebrated Poincaré-Birkhoff theorem. We write the proof of this theorem, by using positive paths, and the proof of its generalization due to P. Carter. We will also explain the links with the free disk chains introduced in the subject by J. Franks. We will finish the paper by studying the local versions where the upper curve is not invariant and will explain why this curve or its image must be a graph to get such a generalization.
\end{abstract}

\section{INTRODUCTION}

In 1913, Birkhoff Bi1 proved a celebrated result, conjectured and proved in special cases by Poincaré $[\mathrm{P}$, that asserts that an area-preserving homeomorphism of the closed annulus that satisfies some "twist condition" admits at least two fixed points. Its proof used an index argument that was valid to find one fixed point but was incorrect to get the second one. A small modification of the argument was necessary, and Birkhoff corrected this minor error in a paper Bi2 published in 1925 (see also the well-detailed expository paper of Brown and Newman [BN]), where he made some generalizations: the area-preserving hypothesis was replaced by a topological intersection property, and the hypothesis about the invariance of the annulus was weakened. A generalization of the Poincaré-Birkhoff theorem, with a nicer intersection property, was obtained by Carter [C]. Looking at noninvariant annuli, which are useful in the study of nonautonomous planar Hamiltonian systems to show the existence of periodic orbits, further generalizations have been obtained by Jacobowitz [J1, J2, Ding [D] and other authors (see the paper of Dalbono and Rebelo DR on the subject).

More recently, Franks introduced new ideas by giving a proof of the PoincaréBirkhoff theorem that uses Brouwer's lemma on translation arcs (Kerékjártó already observed the link between Brouwer's theory and the Poincaré-Birkhoff theorem $[\mathrm{K}]$ ). His proof uses only the fact that the map has no wandering point, and the twist property can be extended to other interesting situations. His ideas have been developed by many authors.

Received by the editors May 28, 2009, and, in revised form, July 2, 2009.

2000 Mathematics Subject Classification. Primary 37E30, 37E40, 37J10.

Key words and phrases. Fixed point, boundary twist condition, positive path.

The authors have been supported by ANR (Symplexe, ANR-06-BLAN-0030-01) and the project 111-2-01.

(C)2009 American Mathematical Society Reverts to public domain 28 years from publication 
The goal of this paper is to introduce the notion of a positive path of a homeomorphism, which seems to be a natural object for understanding Birkhoff's arguments. In the first section we will give a proof of the (classical) Poincaré-Birkhoff theorem using existence of positive paths that cross the annulus. In the second section we will make a link with Franks' ideas. We will recall a natural construction, in the spirit of Franks' ideas, and will observe that it permits us to obtain positive paths that cross the annulus and so to get the Poincaré-Birkhoff theorem without the help of Brouwer's theory. This method stays valid to prove Carter's theorem in the case of an invariant annulus. We will see in the last section that the arguments may be generalized in the situation where the annulus is not invariant, and so we will give a new proof of Carter's theorem in its generality.

\section{Statement and proof of the Poincaré-Birkhoff theorem}

In what follows, a path on a topological space $X$ is a continuous map $\gamma: I \rightarrow X$ defined on a segment $I=[a, b] \subset \mathbf{R}$. The origin and the extremity of $\gamma$ are respectively $\gamma(a)$ and $\gamma(b)$. If $X_{1}$ and $X_{2}$ are two subsets of $X$, we will say that $\gamma$ joins $X_{1}$ to $X_{2}$ if its origin belongs to $X_{1}$ and its extremity belongs to $X_{2}$. The restriction of $\gamma$ to a compact interval $J \subset I$ is a subpath of $\gamma$. If $\gamma$ is one-to-one, $\gamma$ is an arc; if $\gamma(a)=\gamma(b)$, it is a loop; if $\gamma(a)=\gamma(b)$ and $\gamma$ is one-to-one on $[a, b)$, it is a simple loop. The concatenation of two paths (when it is defined) is denoted by $\gamma_{1} \gamma_{2}$. As is usually done, we often will not make any distinction between a path and its image. In particular, if $Y$ is a subset of $X$, we will write $\gamma \subset Y$ if the image of $\gamma$ is included in $Y$.

We write $\mathbf{T}^{1}=\mathbf{R} / \mathbf{Z}$ and we fix in this section and the next one a homeomorphism $F$ of $\mathbf{A}=\mathbf{T}^{1} \times[0,1]$ homotopic to the identity and a lift $f$ of $F$ to the universal cover $\widetilde{\mathbf{A}}=\mathbf{R} \times[0,1]$. We suppose that $f$ satisfies the boundary twist condition:

$$
\text { for every } x \in \mathbf{R}, p_{1} \circ f(x, 0)<x<p_{1} \circ f(x, 1) \text {, }
$$

where $p_{1}: \widetilde{\mathbf{A}} \rightarrow \mathbf{R}$ is the first projection. We write $\operatorname{Fix}_{*}(F)$ for the set of fixed points of $F$ that are lifted to fixed points of $f$. Let us state the Poincaré-Birkhoff theorem.

Theorem 1. If $F$ preserves the measure induced by $d x \wedge d y$, then $\sharp \operatorname{Fix}_{*}(F) \geq 2$.

Let us recall the ideas of Birkhoff. The vector field $\widetilde{X}: z \mapsto f(z)-z$ is invariant under the covering automorphism $T:(x, y) \mapsto(x+1, y)$ and lifts a vector field $X$ on $\mathbf{A}$ whose singular set is exactly $\operatorname{Fix}_{*}(F)$. If $\gamma$ is a path in $\widetilde{\mathbf{A}} \backslash \operatorname{Fix}(f)$, one may define the variation of angle

$$
i_{\gamma} f=\int_{\widetilde{X} \circ \gamma} d \theta,
$$

where

$$
d \theta=\frac{1}{2 \pi} \frac{x d y-y d x}{x^{2}+y^{2}}
$$

is the usual polar form on $\mathbf{R}^{2} \backslash\{0\}$. The form $d \theta$ being closed can be integrated on any (even nonsmooth) path in $\mathbf{R}^{2} \backslash\{0\}$. The theorem will be proved if one finds a loop $\Gamma$ in $\widetilde{\mathbf{A}} \backslash \operatorname{Fix}(f)$ such that $i_{\Gamma} \neq 0$. Indeed if $\operatorname{Fix}_{*}(F)$ is finite (equivalently if $\operatorname{Fix}(f)$ is discrete), then

$$
i_{\Gamma} f=\sum_{z \in \operatorname{Fix}(f)} i(\tilde{X}, z) \int_{\xi_{z} \circ \Gamma} d \theta
$$


where $i(\widetilde{X}, z)$ denotes the Poincare index of $\widetilde{X}$ at $z$ and $\xi_{z}$ is the vector field $z^{\prime} \mapsto z^{\prime}-z$. This implies that $\sharp \operatorname{Fix}_{*}(F) \geq 2$ because $i(\widetilde{X}, z)=i(X, \pi(z))$ and because the Poincaré-Hopf formula asserts that

$$
\sum_{z \in \operatorname{Fix}_{*}(F)} i(X, z)=\chi(\mathbf{A})=0 .
$$

If we can find two paths $\gamma$ and $\gamma^{\prime}$ such that $i_{\gamma} f=i_{\gamma^{\prime}} f \neq 0$, the first one joining $\mathbf{R} \times\{0\}$ to $\mathbf{R} \times\{1\}$, the second one joining $\mathbf{R} \times\{1\}$ to $\mathbf{R} \times\{0\}$, then the loop $\Gamma=\gamma \delta \gamma^{\prime} \delta^{\prime}$ obtained by adding horizontal segments on each boundary line will satisfy $i_{\Gamma} f=2 i_{\gamma} f \neq 0$. In Bi1 Birkhoff composes $F$ with a small vertical translation to build such paths. Suppose that the displacement is positive, the iterates by the perturbed map $G$ of $\mathbf{T}^{1} \times\{0\}$ are pairwise disjoint, and they are not all included in the annulus (because $G$ also preserves the area). Birkhoff chooses an arc $\alpha$ that joins a point $z \in \mathbf{T}^{1} \times\{0\}$ to $G(z)$ and by concatenation of the iterates of $\alpha$ constructs an arc that joins $\mathbf{T}^{1} \times\{0\}$ to $\mathbf{T}^{1} \times\{1\}$ and that is lifted into an arc $\gamma$ satisfying $i_{\gamma} f=-\frac{1}{2}$. We will give here a simple construction, in the spirit of Birkhoff's ideas, that does not need any perturbation and that is still valid under a weaker hypothesis than the preservation of the area.

Definition 1. Let $G$ be a homeomorphism of a topological space $X$. A positive path of $G$ is a path $\gamma: I \rightarrow X$ such that for every $t, t^{\prime}$ in $I$,

$$
t^{\prime} \geq t \Rightarrow G\left(\gamma\left(t^{\prime}\right)\right) \neq \gamma(t)
$$

Observe that a positive path $\gamma$ does not meet the fixed point set, that any subpath of $\gamma$ is positive and that the images $G^{k} \circ \gamma, k \in \mathbf{Z}$, are also positive.

Proposition 1. If $\gamma$ is a positive path of $f$ that joins a boundary line of $\widetilde{\mathbf{A}}$ to the other one, then $i_{\gamma} f=-\frac{1}{2}$.

Proof. We write the proof in the case where $\gamma$ joins $\mathbf{R} \times\{0\}$ to $\mathbf{R} \times\{1\}$, the other case being similar. The boundary of the simplex

$$
\Delta=\left\{\left(t, t^{\prime}\right) \in I^{2} \mid t^{\prime} \geq t\right\}
$$

may be written $\partial \Delta=\delta_{d} \delta_{h} \delta_{v}$ where $\delta_{d}$ is the diagonal, $\delta_{h}$ a horizontal segment and $\delta_{v}$ a vertical one. The path $\gamma$ being positive, the map

$$
\Phi:\left(t, t^{\prime}\right) \mapsto f\left(\gamma\left(t^{\prime}\right)\right)-\gamma(t)
$$

does not vanish on $\Delta$, and one has

$$
\int_{\Phi \circ \delta_{d}} d \theta+\int_{\Phi \circ\left(\delta_{h} \delta_{v}\right)} d \theta=\int_{\Phi \circ \partial \Delta} d \theta=0 .
$$

Observe now that the image by $\Phi$ of each segment $\delta_{h}$ and $\delta_{v}$ does not intersect the vertical half-line $\{0\} \times(-\infty, 0]$. This implies that

$$
i_{\gamma} f=\int_{\Phi \circ \delta_{d}} d \theta=-\int_{\Phi \circ\left(\delta_{h} \delta_{v}\right)} d \theta=-\frac{1}{2} .
$$

Recall that a wandering point of a homeomorphism $f$ of a topological space $X$ is a point that admits a wandering neighborhood $U$, which means a neighborhood $U$ such that the $f^{k}(U), k \geq 0$, are pairwise disjoint. Recall that a Urysohn space is a topological space such that two distinct points may be separated by closed neighborhoods. 
Proposition 2. Suppose that $X$ is a connected and locally path-connected Urysohn space and that $G$ is a fixed point free homeomorphism of $X$ with no wandering point. If $Z \subset X$ satisfies $G(Z) \subset Z$, then for every $z \in X$ there exists a positive path of $G$ that joins $Z$ to $z$.

Proof. One must prove the equality $Y=X$, where $Y$ is the set of points that may be joined by a positive path of $G$ whose origin belongs to $Z$. The space $X$ being connected, it is sufficient to prove that $\bar{Y} \subset \operatorname{Int}(Y)$. Fix $z_{0} \in \bar{Y}$. By hypothesis, one can find a path-connected neighborhood $V$ of $z_{0}$ such that $\bar{V} \cap G(\bar{V})=\emptyset$. We will prove that $V \subset Y$.

The fact that $z_{0} \in \bar{Y}$ implies that there exists a positive path $\gamma_{0}: I \rightarrow X$ from $Z$ to $V$. The closures of the subsets $J=\gamma_{0}^{-1}(V)$ and $J^{\prime}=\gamma_{0}^{-1}(G(V))$ do not intersect because $\bar{V} \cap G(\bar{V})=\emptyset$. This implies that $\inf J \neq \inf J^{\prime}$.

Suppose first that inf $J<\inf J^{\prime}$ (this includes the case where $J^{\prime}=\emptyset$ ). In that case, there is a subpath $\gamma_{1}$ of $\gamma_{0}$ from $Z$ to $V$ that does not meet $G(V)$. For every $z \in V$ one can find a path $\gamma$ inside $V$ that joins the extremity $z_{1}$ of $\gamma_{1}$ to $z$. The path $\gamma_{2}=\gamma_{1} \gamma$ is positive because $\gamma_{1}$ is positive and $G(\gamma)$ is disjoint both from $\gamma$ and $\gamma_{1}$. This implies that $z \in Y$.

Suppose now that inf $J^{\prime}<\inf J$. In that case, there is a subpath $\gamma_{1}$ of $\gamma_{0}$ from $Z$ to $G(V)$ that does not meet $V$. We denote by $z_{1}$ its extremity. The point $G\left(z_{1}\right)$ does not belong to $\gamma_{1}$ because this path is positive. The path being compact ( $X$ is Hausdorff), one can find a path-connected neighborhood $U \subset G(V)$ of $z_{1}$ such that $G(U)$ does not intersect $\gamma_{1}$. The set $U$ being nonwandering, one can find a point $z_{2} \in U$ whose positive orbit meets $G^{-1}(U) \subset V$. Choose a path $\gamma$ inside $U$ that joins $z_{1}$ to $z_{2}$. The path $\gamma_{2}=\gamma_{1} \gamma$ does not meet $V$ and is positive because $\gamma_{1}$ is positive and $G(\gamma)$ is disjoint from $\gamma_{1}$ and $\gamma$. Let us consider the integer $k \geq 1$ such that $G^{k}\left(\gamma_{2}\right) \cap V \neq \emptyset$ and $G^{k^{\prime}}\left(\gamma_{2}\right) \cap V=\emptyset$ if $0 \leq k^{\prime}<k$. Since $G(Z) \subset Z$, the path $G^{k}\left(\gamma_{2}\right)$ is a positive path from $Z$ to $V$ that does not meet $G(V)$. We conclude as in the first case.

Remark 1. A classical result asserts that if $\gamma: I \rightarrow X$ is a path in a Hausdorff space $X$, then there exists an arc $\gamma^{\prime}: J \rightarrow X$ joining the origin of $\gamma$ to its extremity such that for every couple $\left(s, s^{\prime}\right) \in J^{2}$ satisfying $s<s^{\prime}$, there exists a couple $\left(t, t^{\prime}\right) \in I^{2}$ such that $\gamma^{\prime}(s)=\gamma(t), \gamma^{\prime}\left(s^{\prime}\right)=\gamma\left(t^{\prime}\right)$ and $t<t^{\prime}$. Therefore in Proposition 2 , one may replace path with arc in the conclusion. For the same reason a locally path-connected space is locally arc-connected. In the case where $X$ is "obviously" arc-connected (e.g. if $X$ is a manifold) one can get directly the improved version of the theorem. In the proof above one may suppose that $\gamma_{0}, \gamma_{1}$ and $\gamma$ are arcs. To construct an $\operatorname{arc} \gamma_{2}$ joining $Z$ to $z_{2}$, one considers the "last point" $z_{3}$ where $\gamma$ meets $\gamma_{1}$. Then one concatenates the subpath of $\gamma_{1}$ that joins its origin to $z_{3}$ and the subpath of $\gamma$ that joins $z_{3}$ to its extremity.

Remark 2. If one applies the proposition with $Z$ equal to the positive orbit of a point $z$, one gets a positive path from a point $G^{k^{\prime}}(z), k^{\prime} \geq 0$, to $G^{-1}(z)$. Therefore, for every point $z$ one may find $k<0$ and a positive arc that joins $z$ to $G^{k}(z)$ (of course $k \neq-1$ ).

Let us explain how to deduce Theorem 1 from Propositions 1 and 2 , 
Proof of Theorem 1. One may suppose that $\operatorname{Fix}_{*}(F)$ does not separate the two boundary circles (otherwise $\sharp \operatorname{Fix}_{*}(F)=+\infty$ ). If $n$ is large enough, the homeomorphism $F^{\prime}$ of the annulus $\mathbf{A}^{\prime}=\mathbf{R} / n \mathbf{Z} \times[0,1]$ lifted by $f$ has no fixed points except for the ones that are lifted to fixed points of $f$. Therefore $\operatorname{Fix}\left(F^{\prime}\right)=\operatorname{Fix}_{*}\left(F^{\prime}\right)$ does not separate the boundary circles of $\mathbf{A}^{\prime}$, and one may consider the connected component $W$ of $\mathbf{A}^{\prime} \backslash \operatorname{Fix}\left(F^{\prime}\right)$ that contains the boundary. Moreover $F^{\prime}$ has no wandering point because it preserves the area. Applying Proposition 2 to $X=W$, to $G=\left.F^{\prime}\right|_{W}$ and to $Z=\mathbf{R} / n \mathbf{Z} \times\{0\}$ or $Z=\mathbf{R} / n \mathbf{Z} \times\{1\}$, one constructs a positive path of $F^{\prime}$ from one of the boundary circles of $\mathbf{A}^{\prime}$ to the other one. Such a path is lifted to a positive path of $f$ from the corresponding boundary line to the other one. Theorem 1 follows from Proposition 1 .

Remark 3. One can prove that $F^{\prime}$ has no wandering point if it is the case for $F$. Indeed, let $T^{\prime}$ be a generator of the (finite) group of automorphisms of the covering space $\mathbf{A}^{\prime}$. The fact that $F$ has no wandering point implies that for every nonempty open set $U \subset \mathbf{A}^{\prime}$, there exists $q \geq 1$ and $p \in \mathbf{Z}$ such that $F^{\prime q}(U) \cap T^{\prime p}(U) \neq \emptyset$. Let us fix a nonempty open set $U_{0} \subset \mathbf{A}^{\prime}$ and define a sequence $\left(U_{k}\right)_{k \geq 0}$ of nonempty open sets where $U_{k+1}$ may be written $U_{k+1}=F^{\prime} q_{k}\left(U_{k}\right) \cap T^{\prime p_{k}}\left(U_{k}\right)$. One deduces that for every $k^{\prime}>k$, one has $U_{k^{\prime}} \subset F^{\prime q_{k}+\ldots q_{k^{\prime}-1}}\left(U_{k}\right) \cap T^{\prime p_{k}+\ldots p_{k^{\prime}-1}}\left(U_{k}\right)$. One can find $k^{\prime}>k$ such that $p_{k}+\cdots+p_{k^{\prime}-1}=0(\bmod n)$. This implies that $U_{k}$ is nonwandering and therefore that $U_{0}$ itself is nonwandering. So Theorem 1 is valid if instead of supposing that $F$ preserves the area, one supposes that $F$ has no wandering point. Anyway, instead of working in a finite cover of $\mathbf{A}$, one can prove directly that the conclusion of Proposition 2 occurs if $X$ is the connected component of $\widetilde{\mathbf{A}} \backslash \operatorname{Fix}(f)$ that contains the boundary, if $G=\left.f\right|_{X}$ and if $Z$ satisfies $f(Z) \subset Z$ and $T(Z)=Z$.

\section{Links With Franks' METHOD}

In [F1, Franks gave a proof of Theorem 1 valid in the case where $F$ has no wandering point and that uses the following result of Brouwer $[\mathrm{Br}]$ : if an orientationpreserving homeomorphism $g$ of $\mathbf{R}^{2}$ has a periodic point of period $q \geq 2$, then one can construct a loop $\Gamma$ such that $i_{\Gamma} g=1$. By a simple perturbation argument, he observed that such a loop still exists if there exists a periodic free disk chain, which means a family $\left(U_{r}\right)_{r \in \mathbf{Z} / n \mathbf{Z}}$ of pairwise disjoint free (i.e., disjoint from their image) topological open disks such that for every $r \in \mathbf{Z} / n \mathbf{Z}$, one of the positive iterates of $U_{r}$ meets $U_{r+1}$. The facts that $F$ has no wandering point and that $f$ satisfies the boundary twist condition permit him to construct such a chain under the condition of finiteness of $\operatorname{Fix}_{*}(F)$. A periodic free disk chain may be obtained under more general twist conditions, which permits Franks to state very useful generalizations of the Poincaré-Birkhoff theorem. We will recall now a natural construction for getting periodic free disk chains and will observe that it gives positive paths that cross the annulus.

Alternate proof of Theorem 1. Here again we suppose that $\operatorname{Fix}_{*}(F)$ does not separate the two boundary circles and write $W$ for the connected component of $\mathbf{A} \backslash$ $\operatorname{Fix}_{*}(F)$ that contains the boundary. Let us consider a triangulation $\mathcal{T}=\left(\sigma_{i}\right)_{i \in I}$ of $W$. Subdividing some simplices if necessary, one may assume that each 2-cell $\sigma_{i}$ is free by $f$ when lifted to $\widetilde{\mathbf{A}}$. 
Lemma 1. For every $i, i^{\prime}$ in $I$, there exists a sequence $\left(\sigma_{i_{k}}\right)_{0 \leq k \leq l}$ such that $\sigma_{i_{0}}=$ $\sigma_{i}, \sigma_{i_{l}}=\sigma_{i^{\prime}}$ and $f\left(\sigma_{i_{k}}\right) \cap \sigma_{i_{k+1}} \neq \emptyset$ if $0 \leq k<l$.

Proof. Fix $i \in I$ and define a subset $J \subset I$ by writing $i^{\prime} \in J$ if there exists a sequence $\left(\sigma_{i_{k}}\right)_{0 \leq k \leq l}$ such that $\sigma_{i_{0}}=\sigma_{i}, \sigma_{i_{l}}=\sigma_{i^{\prime}}$ and $f\left(\sigma_{i_{k}}\right) \cap \sigma_{i_{k+1}} \neq \emptyset$ if $0 \leq k<l$. Observe now that $X=\bigcup_{i^{\prime} \in J} \sigma_{i^{\prime}}$ is a closed subset of $W$ such that $F(X) \subset \operatorname{Int}_{W}(X)$. This implies that $\operatorname{Int}_{W}(X) \backslash F(X)$ is a wandering open set. By hypothesis, one deduces that $F(X)=\operatorname{Int}_{W}(X)$. We deduce that $X$ is a closed and open subset of $W$, which implies by connectedness of $W$ that it is equal to $W$.

This transitivity property and the twist condition could permit us to construct a periodic chain $\left(\widetilde{\sigma}_{i_{r}}\right)_{r \in \mathbf{Z} / n \mathbf{Z}}$ of $f$ made of distinct cells of the lifted triangulation $\left(\widetilde{\sigma}_{i}\right)_{i \in \tilde{I}}$. Guillou and Le Roux [ $\mathrm{L}$ observed that here again, one may find a loop $\Gamma$ such that $i_{\Gamma} f=1$. We will see below how to construct a positive path from $\mathbf{R} \times\{0\}$ to $\mathbf{R} \times\{1\}$. We have found a sequence $\left(\sigma_{i}\right)_{0 \leq i \leq n}$ of free closed disks of $\widetilde{\mathbf{A}}$ whose interiors are pairwise disjoint such that $\sigma_{0} \cap(\mathbf{R} \times\{0\}) \neq \emptyset, \sigma_{n} \cap(\mathbf{R} \times\{1\}) \neq \emptyset$ and $f\left(\sigma_{i}\right) \cap \sigma_{i+1} \neq \emptyset$ for every $i \in\{0, \ldots, n-1\}$. Among all such sequences (the cells are not necessarily cells of the triangulation), choose a sequence $\left(\sigma_{i}\right)_{0 \leq i \leq n}$ for which the integer $n$ is the smallest possible (note that $n \geq 1$ because of the boundary twist condition) and observe that $f^{k}\left(\sigma_{i}\right) \cap \sigma_{j}=\emptyset$ if $j>i+1$ and $k \geq 1$. Indeed suppose that

$$
E=\left\{(i, j, k) \mid j>i+1, \quad k \geq 1, \quad f^{k}\left(\sigma_{i}\right) \cap \sigma_{j} \neq \emptyset\right\}
$$

is not empty and define

$$
\begin{aligned}
& i_{0}=\min \{i \mid \text { there exist } j>i+1, k \geq 1 \text { such that }(i, j, k) \in E\}, \\
& j_{0}=\max \left\{j>i_{0}+1 \mid \text { there exists } k \geq 1 \text { such that }\left(i_{0}, j, k\right) \in E\right\}, \\
& k_{0}=\min \left\{k \geq 1 \mid\left(i_{0}, j_{0}, k\right) \in E\right\} .
\end{aligned}
$$

Observe now that the sequence

$$
\left(f^{k_{0}-1}\left(\sigma_{0}\right), \ldots, f^{k_{0}-1}\left(\sigma_{i_{0}}\right), \sigma_{j_{0}}, \ldots, \sigma_{n}\right)
$$

satisfies the same properties as the sequence $\left(\sigma_{i}\right)_{0 \leq i \leq n}$, contradicting the minimality of $n$. This minimality also implies that $\sigma_{0}$ is the only disk that meets $\mathbf{R} \times\{0\}$ and that $\sigma_{n}$ is the only disk that meets $\mathbf{R} \times\{1\}$.

The set $\bigcup_{0 \leq i \leq n} f^{n-i}\left(\sigma_{i}\right)$ is connected and intersects the two boundary lines. Choose a point $z_{0} \in f^{n}\left(\sigma_{0}\right) \cap(\mathbf{R} \times\{0\})$, a point $z_{n+1} \in \sigma_{n} \cap(\mathbf{R} \times\{1\})$ and for every $i \in\{1, \ldots, n\}$ a point $z_{i} \in f^{n-i+1}\left(\sigma_{i-1}\right) \cap f^{n-i}\left(\sigma_{i}\right)$. Then choose for every $i \in\{0, \ldots, n\}$ an arc $\gamma_{i}$ joining $z_{i}$ to $z_{i+1}$ such that $\left(\gamma_{i} \backslash\left\{z_{i}, z_{i+1}\right\}\right) \subset \operatorname{Int}\left(f^{n-i}\left(\sigma_{i}\right)\right)$. The path $\gamma_{0} \gamma_{1} \ldots \gamma_{n}$ is positive. Indeed suppose that there exists $z \in \gamma_{j}$ such that $f(z) \in \gamma_{i}$, where $i \leq j$. The fact that $\gamma_{j}$ is free implies that $i<j$ and that $z \neq z_{j}$ if $i=j-1$. The fact that $f^{k}\left(\sigma_{i}\right) \cap \sigma_{j}=\emptyset$ if $j>i+1$ and $k \geq 1$ implies that $i \geq j-1$ and that $z \neq z_{j+1}$ if $i=j-1$ (indeed $\left.f\left(\gamma_{j}\right) \cap \gamma_{i} \subset f^{n-j+1}\left(\sigma_{j}\right) \cap f^{n-i}\left(\sigma_{i}\right)\right)$. But if $i=j-1$ and $z \notin\left\{z_{j}, z_{j+1}\right\}$, then $f(z) \in \operatorname{Int}\left(f^{n-j+1}\left(\sigma_{j}\right)\right) \cap f^{n-j+1}\left(\sigma_{j-1}\right)=\emptyset$. Therefore there is no such point $z$.

A slight modification of the argument will give us the following result. The first part of the theorem was already observed in $[\mathrm{Bi2}]$ and $[\mathrm{K}$. The second part is Carter's theorem [C] for an invariant annulus (see also [F2], G1], G2 for proofs that use Brouwer's theory). Recall that a simple loop in $\mathbf{A}$ is essential if it is not null-homotopic. 
Theorem 2. If every essential simple loop meets its image by $F$, then $\sharp \mathrm{Fix}_{*}(F) \geq$ 1 ; if every essential simple loop meets its image by $F$ in at least two points, then $\sharp \operatorname{Fix}_{*}(F) \geq 2$.

Proof. Suppose that $\sharp \operatorname{Fix}_{*}(F) \leq 1$ and keep the same notation. Either there is no positive arc from the lower boundary circle to the upper one or there is no positive arc from the upper boundary circle to the lower one. Assume that we are in the first case and instead of considering a triangulation of $W$ start with a brick decomposition $\mathcal{B}=\left(\sigma_{i}\right)_{i \in I}$. In this dual notion, the bricks are closed disks obtained as the closures of the connected components of the complement in $W$ of a locally finite graph $\Sigma$ whose vertices are locally the extremities of exactly three edges. Here again, one decomposes each brick to get a brick decomposition with bricks that are free when lifted to $\widetilde{\mathbf{A}}$. Define a subset $J \subset I$ by writing $i \in J$ if there exists a sequence $\left(\sigma_{i_{k}}\right)_{0 \leq k \leq l}$ such that $\sigma_{i_{0}} \cap \mathbf{T}^{1} \times\{0\}, \sigma_{i_{l}}=\sigma_{j}$ and $f\left(\sigma_{i_{k}}\right) \cap \sigma_{i_{k+1}} \neq \emptyset$. Here again $X=\bigcup_{j \in J} \sigma_{j}$ is a closed subset of $W$ such that $F(X) \subset \operatorname{Int}_{W}(X)$, and one knows that $X$ is neighborhood of $\mathbf{T}^{1} \times\{0\}$ that does not intersect the upper boundary (otherwise the previous proof will give us a positive path from $\mathbf{T}^{1} \times\{0\}$ to $\mathbf{T}^{1} \times\{1\}$ ). Moreover one knows that $X$ is a subsurface with boundary of $W$ (every union of bricks of a brick decomposition has this property).

In the case where $\operatorname{Fix}_{*}(F)=\emptyset$, then $\partial X$ is compact and may be written as a disjoint union of finitely many simple loops. As $\partial X$ separates the two boundary lines, one of them must be essential. This essential loop is disjoint from its image because $F(\partial X) \subset \operatorname{Int}(X)$.

In the case where $\operatorname{Fix}_{*}(F)=\left\{z_{0}\right\}$, then each connected component of $\partial X$ that is not a simple loop becomes a simple loop when one adds $z_{0}$. Here again, even if there may be infinitely many such loops, one of them must be essential because $\partial X \cup\left\{z_{0}\right\}$ separates the boundary circles. This implies the existence of an essential simple loop that meets its image in fewer than two points.

\section{LOCAL VERSIONS}

We conclude with the versions of the Poincaré-Birkhoff theorem on noninvariant annuli. We fix two essential simple loops $C_{1}$ and $C_{2}$ in $\mathbf{T}^{1} \times(0,+\infty)$ that project injectively onto $\mathbf{T}^{1} \times\{0\}$. We denote by $\mathbf{A}_{i}$ the closed annulus bounded by $\mathbf{T}^{1} \times\{0\}$ and $C_{i}$ and write $\operatorname{Int}\left(\mathbf{A}_{i}\right)=\mathbf{A}_{i} \backslash C_{i}$. We denote by $\widetilde{C}_{i}, \widetilde{\mathbf{A}}_{i}$ and $\operatorname{Int}\left(\widetilde{\mathbf{A}}_{i}\right)$ the respective preimages of $C_{i}, \mathbf{A}_{i}$ and $\operatorname{Int}\left(\mathbf{A}_{i}\right)$ in the universal cover $\mathbf{R} \times[0,+\infty)$. We fix in this section a homeomorphism $F: \mathbf{A}_{1} \rightarrow \mathbf{A}_{2}$ that preserves the orientation and leaves invariant the circle $\mathbf{T}^{1} \times\{0\}$ and a lift $f: \widetilde{\mathbf{A}}_{1} \rightarrow \widetilde{\mathbf{A}}_{2}$ that satisfies the following twist condition:

$$
z \in \mathbf{R} \times\{0\} \Rightarrow p_{1} \circ f(z)<p_{1}(z), \quad z \in \widetilde{C}_{1} \Rightarrow p_{1} \circ f(z)>p_{1}(z) .
$$

Here again we write $\operatorname{Fix}_{*}(F)$ for the set of fixed points of $F$ lifted to fixed points of $f$.

The first result is the version of Theorem 1 on a noninvariant annulus when $F$ is supposed to be area-preserving. Under this condition, it is a particular case of Ding's theorem [D].

Theorem 3. Let us suppose that for every nonempty open set $U \subset \mathbf{A}_{1}$ (resp. $U \subset$ $\left.\mathbf{A}_{2}\right)$, there exists $n>0$ (resp. $\left.n<0\right)$ such that $f^{n}(U) \not \subset \mathbf{A}_{1}$ (resp. $\left.f^{n}(U) \not \subset \mathbf{A}_{2}\right)$ or $f^{n}(U) \cap U \neq \emptyset$. Then $\sharp \operatorname{Fix}_{*}(F) \geq 2$. 

[C].

The second result is stronger; it is the most general version of Carter's theorem

Theorem 4. If every essential simple loop in $\mathbf{A}_{1}$ meets its image by $F$, then $\sharp \operatorname{Fix}_{*}(F) \geq 1$; if every essential simple loop in $\mathbf{A}_{1}$ meets its image by $F$ in at least two points, then $\sharp \operatorname{Fix}_{*}(F) \geq 2$.

As in section 1, if we can find two paths $\gamma$ and $\gamma^{\prime}$ in $\widetilde{\mathbf{A}}_{1} \backslash \operatorname{Fix}(f)$ such that $i_{\gamma} f$ and $i_{\gamma^{\prime}} f$ both belong to $\left(-\frac{3}{4},-\frac{1}{4}\right)$ - the first one joining $\mathbf{R} \times\{0\}$ to $\widetilde{C}_{1}$, the second one joining $\widetilde{C}_{1}$ to $\mathbf{R} \times\{0\}$-then the loop $\Gamma=\gamma \delta \gamma^{\prime} \delta^{\prime}$ obtained by adding "segments" of $\widetilde{C}_{1}$ and $\mathbf{R} \times\{0\}$ will satisfy $i_{\Gamma} f=-1$ and we will deduce as in section 1 that $\sharp \operatorname{Fix}_{*}(F) \geq 2$.

Let us begin with the following results.

Lemma 2. If there is a positive path $\gamma$ of $f$ in $\widetilde{\mathbf{A}}_{1}$ whose origin is on $\mathbf{R} \times\{0\}$ such that $f \circ \gamma$ is not included in $\operatorname{Int}\left(\widetilde{\mathbf{A}}_{1}\right)$, then there is a positive path $\gamma^{\prime}$ in $\widetilde{\mathbf{A}}_{1}$ joining $\mathbf{R} \times\{0\}$ to $\widetilde{C}_{1}$ such that the image of its extremity does not belong to $\operatorname{Int}\left(\widetilde{\mathbf{A}}_{1}\right)$. We obtain a similar result by replacing $f$ with $f^{-1}$ and $\widetilde{C}_{1}$ with $\widetilde{C}_{2}$.

Lemma 3. If $\gamma$ is a positive path of $f$ in $\widetilde{\mathbf{A}}_{1}$ joining $\mathbf{R} \times\{0\}$ to $\widetilde{C}_{1}$ such that the image of its extremity does not belong to $\operatorname{Int}\left(\widetilde{\mathbf{A}}_{1}\right)$, then $i_{\gamma} f \in\left(-\frac{3}{4},-\frac{1}{4}\right)$. Similarly, if $\gamma$ is a positive path of $f^{-1}$ in $\widetilde{\mathbf{A}}_{2}$ joining $\mathbf{R} \times\{0\}$ to $\widetilde{C}_{2}$ such that the inverse image of its extremity does not belong to $\operatorname{Int}\left(\widetilde{\mathbf{A}}_{2}\right)$, then $i_{f^{-1} \gamma} f \in\left(\frac{1}{4}, \frac{3}{4}\right)$.

Proof of Lemma 2. Suppose that $\gamma \in \widetilde{\mathbf{A}}_{1}$ is a positive path of $f$ whose origin is on $\mathbf{R} \times\{0\}$ and such that $f \circ \gamma \not \subset \operatorname{Int}\left(\widetilde{\mathbf{A}}_{1}\right)$. Taking a subpath if necessary, one may suppose that the extremity $z$ of $f(\gamma)$ does not belong to $\operatorname{Int}\left(\widetilde{\mathbf{A}}_{1}\right)$. Since $C_{1}$ projects injectively on $\mathbf{T}^{1} \times\{0\}$, the vertical arc $\gamma_{v}$ joining $z$ to $\widetilde{C}_{2}$ does not intersect $\widetilde{\mathbf{A}}_{1}$ if $z \notin \widetilde{C}_{1}$ and intersects $\widetilde{\mathbf{A}}_{1}$ only at $z$ if $z \in \widetilde{C}_{1}$. This implies that $\gamma^{\prime}=\gamma f^{-1}\left(\gamma_{v}\right)$ is a positive path in $\widetilde{\mathbf{A}}_{1}$ joining $\mathbf{R} \times\{0\}$ to $\widetilde{C}_{1}$ and that the image by $f$ of its extremity does not belong to $\operatorname{Int}\left(\widetilde{\mathbf{A}}_{1}\right)$.

Proof of Lemma 3. Let $\gamma \in \widetilde{\mathbf{A}}_{1}$ be a positive path of $f$ joining $\mathbf{R} \times\{0\}$ to $\widetilde{C}_{1}$ such that the image of its extremity does not belong to $\operatorname{Int}\left(\widetilde{\mathbf{A}}_{1}\right)$. We keep the same notation as in Proposition 1. The map $\Phi$ does not reach the vertical $\{0\} \times(-\infty, 0]$, either on $\delta_{v}$ or on $\delta_{h}$. This implies that $i_{\gamma} f \in\left(-\frac{3}{4},-\frac{1}{4}\right)$. Similarly, if $\gamma \in \widetilde{\mathbf{A}}_{2}$ is a positive path of $f^{-1}$ whose origin is on $\mathbf{R} \times\{0\}$ and such that $f^{-1} \circ \gamma$ is not in $\operatorname{Int}\left(\widetilde{\mathbf{A}}_{2}\right)$, then one has $i_{f^{-1}(\gamma)} f=i_{\gamma} f^{-1} \in\left(\frac{1}{4}, \frac{3}{4}\right)$.

Proof of Theorem 3. More precisely, in the case where $\operatorname{Fix}_{*}(F)$ does not separate the two boundary components of $\mathbf{A}_{1}$, we will prove the existence of a positive path of $f$ that joins $\mathbf{R} \times\{0\}$ to $\widetilde{C}_{1}$ such that the image by $f$ of its extremity is not in $\operatorname{Int}\left(\widetilde{\mathbf{A}}_{1}\right)$, and of course we will get a similar result with $f^{-1}$ instead of $f$ and $\widetilde{\mathbf{A}}_{2}$ instead of $\widetilde{\mathbf{A}}_{1}$. Taking a finite cover of $\mathbf{A}_{1}$ if necessary, one may suppose that $\operatorname{Fix}_{*}(F)=\operatorname{Fix}(F)$ and write $W=\mathbf{A}_{1} \backslash \operatorname{Fix}(F)$.

Now look at the proof of Proposition 2 in the case where $X=W, G=\left.F\right|_{W}$ and $Z=\mathbf{T}^{1} \times\{0\}$ and keep the same notation (it makes sense to define the neighborhood $V \subset W)$. The first situation is similar, and we conclude that $V \subset Y$. In the second situation, if $F\left(z_{1}\right) \notin \operatorname{Int}\left(\mathbf{A}_{1}\right)$, we are done by Lemmas 2 and 3 , If not, $U$ can 
be defined similarly, and by hypothesis the point $z_{2}$ can be chosen such that its positive orbit either meets $F^{-1}(V)$ or is not included in $\operatorname{Int}\left(\mathbf{A}_{1}\right)$. If one of the positive iterates of $\gamma_{2}$ is not included in $\operatorname{Int}\left(\mathbf{A}_{1}\right)$, then we conclude using Lemmas 2 and 3 , otherwise the argument is similar to the one in Proposition 2, and we conclude that $V \subset Y$. Note now that if $Y=W$, we can find a positive $\operatorname{arc} \gamma$ of $F$ in $\mathbf{A}_{1}$ from $\mathbf{T}^{1} \times\{0\}$ to $C_{1}$ such that $F \circ \gamma$ is not in $\operatorname{Int}\left(\mathbf{A}_{1}\right)$. Otherwise $\mathbf{A}_{2} \subset \operatorname{Int}\left(\mathbf{A}_{1}\right)$, which contradicts the hypothesis.

Proof of Theorem 4. We suppose that $\operatorname{Fix}_{*}(F)<2$. Replacing $f$ with $f^{-1}$ if necessary, let us assume that there is no positive path that joins $\mathbf{R} \times\{0\}$ to $\widetilde{C}_{1}$ such that the image by $f$ of its extremity is not in $\operatorname{Int}\left(\widetilde{\mathbf{A}}_{1}\right)$. This implies that there is no sequence $\left(\delta_{i}\right)_{0 \leq i \leq n}$ of free closed disks of $\widetilde{\mathbf{A}}_{1} \backslash \operatorname{Fix}(f)$ whose interiors are pairwise disjoint such that $\delta_{0} \cap(\mathbf{R} \times\{0\}) \neq \emptyset, f\left(\delta_{n}\right) \not \subset \operatorname{Int}\left(\widetilde{\mathbf{A}}_{1}\right)$ and $f\left(\delta_{i}\right) \cap \delta_{i+1} \neq \emptyset$ for every $i \in\{0, \ldots, n-1\}$. Indeed if $n$ is minimal among such sequences, then $f^{k}\left(\delta_{i}\right) \subset \operatorname{Int}\left(\widetilde{\mathbf{A}}_{1}\right)$ for every integer $k \geq 1$, if $0 \leq i<n$, and the positive iterates of $\delta_{i}$ are all defined. So the argument of section 2 is still valid: one knows that $f^{k}\left(\delta_{i}\right) \cap \delta_{j}=\emptyset$ if $j>i+1$ and $k \geq 1$. Choose $z_{n+1} \in \delta_{n}$ such that $f\left(z_{n+1}\right) \notin \operatorname{Int}\left(\widetilde{\mathbf{A}}_{1}\right)$ and do the same construction as in section 2 . We will get a positive path in $\widetilde{\mathbf{A}}_{1}$ whose origin is on $\mathbf{R} \times\{0\}$ such that its image by $f$ is not included in $\operatorname{Int}\left(\widetilde{\mathbf{A}}_{1}\right)$. This would contradict Lemma 2 .

Let us consider now a brick decomposition of $W=\mathbf{A}_{1} \backslash \mathrm{Fix}_{*}(F)$ whose bricks are free when lifted to $\widetilde{\mathbf{A}}_{1}$ and define a set $J \subset I$ by the same formula as in the proof of section 2. By the remark just above, one knows that $f\left(\delta_{j}\right) \subset \operatorname{Int}\left(\mathbf{A}_{1}\right)$ for every $j \in J$. We deduce that the subsurface $X=\bigcup_{j \in J} \delta_{j}$ satisfies $F(X) \subset \operatorname{Int}_{W}(X)$, and we can conclude as in the proof of Theorem 2 .

Remark 4 . We have used the fact that the loops $C_{1}$ and $C_{2}$ project injectively onto $\mathbf{T}^{1} \times\{0\}$ only in the proofs of Lemma 2 and Lemma 3. As we will see below, these lemmas are still valid if one of the loops $C_{1}$ or $C_{2}$ projects injectively onto $\mathbf{T}^{1} \times\{0\}$. Therefore, both Theorems 3 and 4 are still valid under this weaker assumption. This was already known ([D, G1]). The proof of Lemma 2 is valid without any assumption on $C_{1}$ and $C_{2}$ : one may find an arc $\gamma_{v}$ from $z$ to $\widetilde{C}_{2}$ included in $\widetilde{\mathbf{A}}_{2}$ that does not meet $\widetilde{\mathbf{A}}_{1}$ if $z \notin \widetilde{C}_{1}$ and that meets $\widetilde{\mathbf{A}}_{1}$ only at $z$ if $z \in \widetilde{C}_{1}$ (but it will not necessarily be vertical if $C_{1}$ does not project injectively on $\left.\mathbf{T}^{1} \times\{0\}\right)$.

The first statement of Lemma 3 is valid if $C_{1}$ projects injectively on $\mathbf{T}^{1} \times\{0\}$ with exactly the same proof. Due to the symmetry of the problem, we still have to prove that the first statement of Lemma 3 is also true if $C_{2}$ projects injectively on $\mathbf{T}^{1} \times\{0\}$. Let $\gamma:[0,1] \rightarrow \widetilde{\mathbf{A}}_{1}$ be a positive path satisfying the hypothesis of the lemma. Modifying $\gamma$ in the uniform topology (the property of being positive is obviously stable by perturbation), one may suppose that $\gamma(t) \notin \mathbf{R} \times\{0\}$ if $t \neq 0$ and $\gamma(t) \notin \widetilde{C}_{1}$ if $t \neq 1$. One cannot compute $i_{\gamma} f$ directly using the arguments of Proposition 1. Indeed the map $\Phi$ does not reach the vertical $\{0\} \times(-\infty, 0]$ on $\delta_{v}$, but it may reach it on $\delta_{h}$. One needs an indirect argument. Write $\widetilde{C}_{2}$ as the graph of a continuous function $\varphi: \mathbf{R} \rightarrow(0,+\infty)$ and define, for every $r \in \mathbf{R}$, the 
homeomorphism

$$
\begin{aligned}
T_{r}: \widetilde{\mathbf{A}}_{2} & \rightarrow \widetilde{\mathbf{A}}_{2}, \\
(x, s \varphi(x)) & \mapsto(x+r, s \varphi(x+r)),
\end{aligned}
$$

where $s \in[0,1]$. Then consider the map

$$
\begin{aligned}
\Psi: \Delta \times[0,+\infty[ & \rightarrow \mathbf{R}^{2}, \\
\left(t, t^{\prime}, r\right) & \mapsto T_{-\left(1-t^{\prime}\right) r} \circ f\left(\gamma\left(t^{\prime}\right)\right)-f^{-1} \circ T_{-r} \circ f(\gamma(t)) .
\end{aligned}
$$

This map may vanish on $\Delta \times[0,+\infty[$. However, observe that $\Psi(t, 1, r) \neq 0$ if $t \neq 1$ (because $f(\gamma(1)) \notin \operatorname{Int}\left(\widetilde{\mathbf{A}}_{1}\right)$ and $\left.f^{-1} \circ T_{-r} \circ f(\gamma(t)) \in \operatorname{Int}\left(\widetilde{\mathbf{A}}_{1}\right)\right)$, that $\Psi\left(0, t^{\prime}, r\right) \in$ $\mathbf{R} \times(0,+\infty)$ if $t^{\prime} \neq 0$ (because $\left.\gamma\left(t^{\prime}\right) \notin \mathbf{R} \times\{0\}\right)$, that $\Psi(0,0, r) \in(-\infty, 0) \times\{0\}$ (because of the twist condition on the lower boundary), that $\Psi(1,1, r) \in(0,+\infty) \times$ $\mathbf{R}$ (because of the twist condition on the upper boundary) and finally that there exists $r_{0} \geq 0$ such that $\Psi(t, 1, r) \in(0,+\infty) \times \mathbf{R}$ if $r \geq r_{0}$ (for obvious reasons).

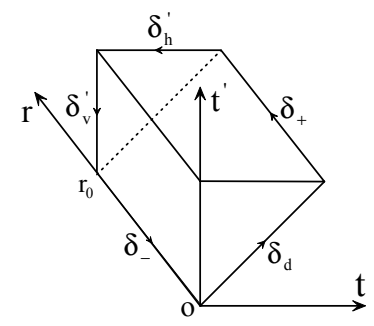

FiguRE 1. Integral illustration

Write (see Figure 1)

- $\delta_{d}$ for the segment joining $(0,0,0)$ to $(1,1,0)$;

- $\delta_{+}$for the segment joining $(1,1,0)$ to $\left(1,1, r_{0}\right)$;

- $\delta_{h}^{\prime}$ for the segment joining $\left(1,1, r_{0}\right)$ to $\left(0,1, r_{0}\right)$;

- $\delta_{v}^{\prime}$ for the segment joining $\left(0,1, r_{0}\right)$ to $\left(0,0, r_{0}\right)$;

- $\delta_{-}$for the segment joining $\left(0,0, r_{0}\right)$ to $(0,0,0)$.

Observe that the loop $\delta_{d} \delta_{+} \delta_{h}^{\prime} \delta_{v}^{\prime} \delta_{-}$bounds a disk where $\Psi$ does not vanish. This implies that

$$
i_{\gamma} f=\int_{\Psi \circ \delta_{d}} d \theta=-\int_{\Psi \circ\left(\delta_{+} \delta_{h}^{\prime} \delta_{v}^{\prime} \delta_{-}\right)} d \theta,
$$

but the last integral belongs to $\left(\frac{1}{4}, \frac{3}{4}\right)$ because $\Psi$ does not reach the vertical $\{0\} \times$ $(-\infty, 0]$ on $\delta_{+} \delta_{h}^{\prime} \delta_{v}^{\prime} \delta_{-}$.

Lemma 3 may fail if none of the loops project injectively onto $\mathbf{T}^{1} \times\{0\}$, unlike what is said in [D] and [J1, and then Theorems [3] and 4 are no longer valid (see also [MU]). We will conclude with the construction of an area-preserving and fixed point free homeomorphism satisfying a twist condition.

Let us consider the polygon $\Pi$ whose vertices are (see Figure 2)

$$
P=(1,0), \quad A=(1,1), \quad B=\left(\frac{1}{2}, 1\right), T^{-1}(A)=(0,1), T^{-1}(P)=(0,0),
$$




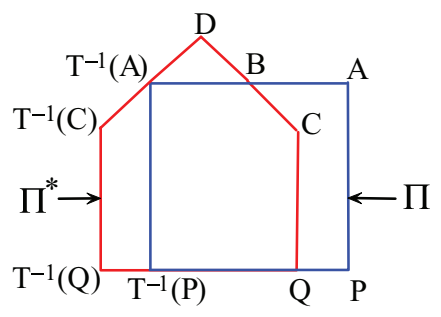

Figure 2. The construction of $f_{0}$

and the polygon $\Pi^{*}$ whose vertices are

$$
Q=\left(\frac{3}{4}, 0\right), C=\left(\frac{3}{4}, \frac{3}{4}\right), \quad D=\left(\frac{1}{4}, \frac{5}{4}\right), T^{-1}(C)=\left(-\frac{1}{4}, \frac{3}{4}\right), T^{-1}(Q)=\left(-\frac{1}{4}, 0\right) .
$$

The bounded domains $U$ and $U^{*}$ enclosed by $\Pi$ and $\Pi^{*}$ having the same area, the piecewise affine map from $\Pi$ to $\Pi^{*}$, sending $P$ on $Q, A$ on $C, B$ on $D, T^{-1}(A)$ on $T^{-1}(C), T^{-1}(P)$ on $T^{-1}(Q)$ and affine on each edge of $\Pi$, may be extended to an area-preserving homeomorphism from $\bar{U}$ to $\bar{U}^{*}$ (this is a consequence of Schoenflies theorem and of a classical result of Oxtoby-Ulam about topologically equivalent measures; see for example the appendix of [BCL]). This homeomorphism may be naturally extended to an area-preserving homeomorphism

$$
f_{0}: \widetilde{\mathbf{A}}_{1}=\bigcup_{k \in \mathbf{Z}} T^{k}(\bar{U})=\mathbf{R} \times[0,1] \rightarrow \widetilde{\mathbf{A}}_{2}=\bigcup_{k \in \mathbf{Z}} T^{k}\left(\bar{U}^{*}\right)
$$

that commutes with $T$. Let us fix $\varepsilon \in(0,1)$ and $M>0$ such that

$$
\begin{cases}p_{1} \circ f_{0}(z)<p_{1}(z) & \text { for every } z \in \mathbf{R} \times[1-\varepsilon, 1] \\ p_{1} \circ f_{0}(z)<p_{1}(z)+M & \text { for every } z \in \mathbf{R} \times[0,1] .\end{cases}
$$

The map

$$
f_{1}:(x, y) \mapsto f_{0}\left(x+\frac{M}{\varepsilon}(y-1), y\right)
$$

coincides with $f_{0}$ on $\mathbf{R} \times\{1\}$, is a translation on $\mathbf{R} \times\{0\}$, preserves the area, commutes with $T$ and satisfies

$$
p_{1} \circ f_{1}(z)<p_{1}(z) \quad \text { for every } z \in \mathbf{R} \times[0,1] .
$$

As a consequence $f_{1}$ has no fixed point. Observe that it sends $E=\left(\frac{3}{4}, 1\right)$ on $B$ and $F=\left(\frac{1}{4}, 1\right)$ on $T^{-1}(A)$.

Now, let us consider (see Figure 3) :

-- the polygon $\Pi_{1}$ whose vertices are $A, E, B, C$;

-- the polygon $\Pi_{2}$ whose vertices are $B, D, T^{-1}(A), F$;

-- $\quad$ the polygon $\Pi_{3}$ whose vertices are $Q, C, B, F, T^{-1}(A), T^{-1}(C), T^{-1}(Q)$; and write $U_{i}$ for the bounded domain enclosed by $\Pi_{i}$.

Similarly let us consider

-- the polygon $\Pi_{1}^{\prime}$ whose vertices are $A^{\prime}, E^{\prime}, B^{\prime}, C^{\prime}$;

-- the polygon $\Pi_{2}^{\prime}$ whose vertices are $B^{\prime}, D^{\prime}, T^{-1}\left(A^{\prime}\right), F^{\prime}$;

-- the polygon $\Pi_{3}^{\prime}$ whose vertices are $Q^{\prime}, C^{\prime}, B^{\prime}, F^{\prime}, T^{-1}\left(A^{\prime}\right), T^{-1}\left(C^{\prime}\right), T^{-1}\left(Q^{\prime}\right)$, 

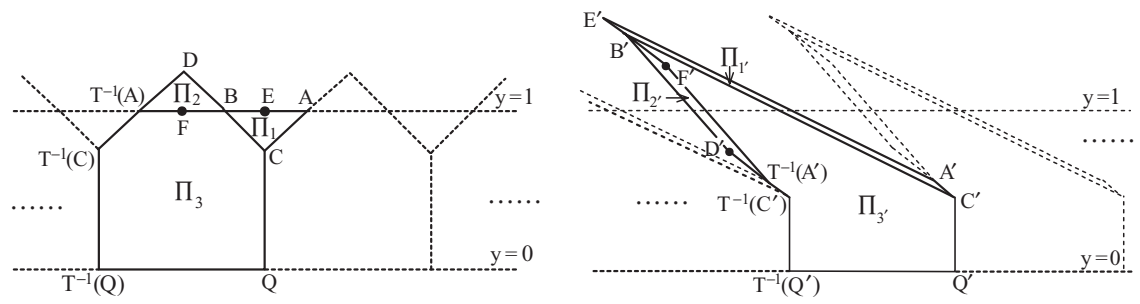

Figure 3. The construction of $h$

where $A^{\prime}=\left(\frac{9}{8}, \frac{35}{64}\right), B^{\prime}=\left(-\frac{3}{4}, \frac{93}{64}\right), C^{\prime}=\left(\frac{5}{4}, \frac{29}{64}\right), D^{\prime}=\left(-\frac{1}{8}, \frac{47}{64}\right), E^{\prime}=\left(-\frac{7}{8}, \frac{99}{64}\right)$, $F^{\prime}=\left(-\frac{1}{2}, \frac{81}{64}\right), Q^{\prime}=\left(\frac{5}{4}, 0\right)$. The bounded domains $U_{1}^{\prime}, U_{2}^{\prime}, U_{3}^{\prime}$ enclosed respectively by $\Pi_{1}^{\prime}, \Pi_{2}^{\prime}, \Pi_{3}^{\prime}$ are pairwise disjoint, and a simple computation gives us

$$
\mu\left(U_{1}^{\prime}\right)=\mu\left(U_{2}^{\prime}\right)=\mu\left(U_{1}\right)=\mu\left(U_{2}\right)=\frac{1}{16}, \mu\left(U_{3}^{\prime}\right)=\mu\left(U_{3}\right)=\frac{7}{8} .
$$

Note that the second coordinate of the middle point of the diagonals of $\Pi_{1}^{\prime}$ and $\Pi_{2}^{\prime}$ is 1 .

The piecewise affine map $\bigcup_{1 \leq i \leq 3} \Pi_{i} \rightarrow \bigcup_{1 \leq i \leq 3} \Pi_{i}^{\prime}$ sending each vertex $X$ on the vertex $X^{\prime}$ may be extended first to an area-preserving homeomorphism $\bigcup_{1 \leq i \leq 3} \overline{U_{i}}$ $\rightarrow \bigcup_{1 \leq i \leq 3} \overline{U_{i}^{\prime}}$ and then to an area-preserving homeomorphism

$$
h: \bigcup_{k \in \mathbf{Z}} T^{k}\left(\bigcup_{1 \leq i \leq 3} \overline{U_{i}}\right) \rightarrow \bigcup_{k \in \mathbf{Z}} T^{k}\left(\bigcup_{1 \leq i \leq 3} \overline{U_{i}^{\prime}}\right)
$$

that commutes with $T$. The homeomorphism

$$
f_{2}: h \circ f_{1} \circ h^{-1}: h\left(\widetilde{\mathbf{A}}_{1}\right) \rightarrow h\left(\widetilde{\mathbf{A}}_{2}\right)
$$

preserves the area, commutes with $T$ and is fixed point free. Nevertheless it satisfies the twist condition. Indeed one has $p_{1} \circ f_{2}(z)=p_{1} \circ f_{1}(z)<p_{1}(z)$ for every $z \in \mathbf{R} \times\{0\}$ and $p_{1}\left(f_{2}(z)\right)>p_{1}(z)$ for every $z$ on the upper boundary $\widetilde{C}_{1}$ of $h\left(\widetilde{\mathbf{A}}_{1}\right)$. Indeed, the last property is true for each vertex $A^{\prime}, E^{\prime}, B^{\prime}, F^{\prime}, T^{-1}\left(A^{\prime}\right)$ (respectively sent onto $\left.C^{\prime}, B^{\prime}, D^{\prime}, T^{-1}\left(A^{\prime}\right), T^{-1}\left(C^{\prime}\right)\right)$, and the map is affine between two vertices.

To get a smooth example, one considers a smooth essential simple loop $C_{1}^{\prime} \subset$ $\pi\left(h\left(\widetilde{\mathbf{A}}_{1}\right)\right)$ very close to $C_{1}=\pi\left(\widetilde{C}_{1}\right)$. Then, on the annulus bounded by $\mathbf{T}^{1} \times\{0\}$ and $C_{1}^{\prime}$, one approximates the diffeomorphism $F_{2}$ lifted by $f_{2}$ by a smooth areapreserving diffeomorphism.

\section{REFERENCES}

[BCL] F. Béguin, S. Crovisier, F. Le Roux : Pseudo-rotations of the open annulus, Bull. Braz. Math. Soc. (N.S.), 37 (2006), 275-306. MR2266384(2008b:37074)

[Bi1] G. D. Birkhoff : Proof of Poincaré's last geometric theorem, Trans. Amer. Math. Soc., 14 (1913), 14-22.

[Bi2] G. D. Birkhoff : An extension of Poincaré's last geometric theorem, Acta. Math., 47 (1925), 297-311.

[Br] L. E. J. Brouwer : Beweis des ebenen Translationssatzes, Math. Ann., 72 (1912), 37-54. MR1511684

[BN] L. E. J. M. Brown, W. D. Newmann : Proof of the Poincaré-Birkhoff fixed point theorem, Michigan. Math. J., 24 (1977), 21-31. MR0448339 (56:6646)

[C] P. H. Carter : An improvement of the Poincaré-Birkhoff fixed point theorem, Trans. Amer. Math. Soc., 269 (1982), 285-299. MR637039 (84h:54041) 
[DR] F. Dalbono, C. Rebelo : Poincaré-Birkhoff fixed point theorem and periodic solutions of asymptotically linear planar Hamiltonian systems, Rend. Sem. Math. Univ. Pol. Torino, 60 (2002), 233-263 (2003). MR2010410(2004j:37100)

[D] W.-Y. Ding : A generalization of the Poincaré-Birkhoff theorem, Proc. Amer. Math. Soc., 88 (1983), 341-346. MR695272 (84f:54053)

[F1] J. Franks : Generalizations of the Poincaré-Birkhoff theorem, Annals of Math. (2), 128 (1988), 139-151. MR951509 (89m:54052)

[F2] J. Franks : A variation on the Poincaré-Birkhoff Theorem, Contemp. Math., 81, Amer. Math. Soc., Providence, RI, 1988, 111-117. MR.986260 (90e:58095)

[G1] L. Guillou : Théorème de translation plane de Brouwer et généralisations du théorème de Poincaré-Birkhoff, Topology, 33 (1994), 331-351. MR1273787 (95h:55003)

[G2] L. Guillou : A simple proof of P. Carter's theorem, Proc. Amer. Math. Soc., 125 (1997), 1555-1559. MR 1372031 (97g:54055)

[J1] H. Jacobowitz : Periodic solutions of $x^{\prime \prime}+f(x, t)=0$ via the Poincaré-Birkhoff theorem, J. Diff. Equations, 20 (1976), 37-52. MR0393673 (52:14482)

[J2] H. Jacobowitz : Corrigendum: The existence of the second fixed point: a correction to "Periodic solutions of $x^{\prime \prime}+f(x, t)=0$ via the Poincaré-Birkhoff theorem", J. Diff. Equations, 25 (1977), 148-149. MR0437857 (55:10778)

[K] B. de Kerékjártó : The plane translation theorem of Brouwer and the last geometric theorem of Poincaré, Acta Sci. Math. Szeged, 4 (1928-29), 86-102.

[L] F. Le Roux : Homéomorphismes de surfaces: théorèmes de la fleur de Leau-Fatou et de la variété stable, Astérisque, 292 (2004). MR2068866 (2005e:37091)

[MU] R. Matins, A. J. Ureña : The star-shaped condition on Ding's version of the PoincaréBirkhoff theorem, Bull. London Math. Soc., 39 (2007), no. 5, 803-810. MR2365229 (2008k:54061)

[P] H. Poincaré : Sur un théorème de géométrie, Rend. Circ. Mat. Palermo, 33 (1912), 375407.

Institut de Mathématiques de Jussieu, Unité Mixte de Recherche 7586, Centre National de la Recherche Scientifique, Université Pierre et Marie Curie, 175 rue du Chevaleret, 75013 Paris, France

E-mail address: lecalvez@math.jussieu.fr

Department of Mathematics, Tsinghua University, Beijing 100084, People's Republic OF CHINA

E-mail address: wjian05@mails.tsinghua.edu.cn

Current address: Laboratoire Analyse Géométrie et Applications, Unité Mixte de Recherche 7539, Centre National de la Recherche Scientifique, Université Paris 13, 93430 Villetaneuse, France

E-mail address: wangjian@math.univ-paris13.fr 